\title{
Enfermedad hidatídica diseminada con localización mediastinal como causa de síndrome de vena cava superior.
}

\author{
PINTO VALDIVIA José Luis*, DE SOSA Yaneth**, GAYOSO CERVANTES Oscar***, DÍAZ URTEAGA Javier,\& \\ CHINCHA Omayra.
}

\section{SUMMARY}

Hydatidic illness is a frequent parasitic zoonosis in our country whose more frequent localization is the liver and the lungs. However other many sites have been described, depending their symptoms on the cyst's size, localization and effect on next structures, being sometimes its evolution fatal.

This is a 52 year-old female patient admitted at emergency room for dysfunction of sensory and neurological focalization. She had a previous history of surgery many years ago by abdominal tumor. We found a collateral circulation on right hemithorax and facial and arms edema at exam. The cerebral CT showed extensive left parietal parenchymal hemorrhage. Thoracoabdominal CT revealed multiple cystic masses of diverse sizes in the mediastinum, lungs, liver and other places. The patient's evolution was torpid, the neurological compromise didn't improve in spite of the treatment against the intracranial hypertension (EH), being also added a nosocomial pneumonia. The patient died after several days. Necropsy revealed presence of cystic lesions in mediastinum, heart's great vessels, pericardium, lungs, liver, spleen and omentum, as well as intra and extraparenchymal hemorrhages of hypertensive cause in brain, not being cystic lesions in central nervous system. We concluded that it was disseminated hydatidic illness, cysts in the mediastinum caused compression of the heart's great vessels, causing a superior cave vein syndrome. ( Rev Med Hered 2001; 12: 142-149).

KEY WORDS: Hydatidic disease, superior cave vein syndrome, intracranial hypertension.

\section{INTRODUCCIÓN}

La enfermedad hidatídica es una zoonosis parasitaria causada por la ingestión de huevos del Echinococcus granulosus. Los huevos eclosionan en el duodeno y los embriones liberados penetran la mucosa del intestino y se diseminan por los sistemas venoso y linfático, pudiendo afectar cualquier parte del cuerpo, siendo el hígado (60\%) y luego los pulmones (30\%) los órganos más comúnmente afectados. Sin embargo se han descrito todas las localizaciones (cerebro, bazo, riñones, corazón, mamas, próstata, páncreas, músculos, huesos, mediastino), dependiendo los síntomas del tamaño del quiste, su localización y afección de estructuras próximas, con complicaciones potencialmente fatales $(1-4)$.

\footnotetext{
* Médico-residente de Gastroenterología. Universidad Peruana Cayetano Heredia. Hospital Nacional Cayetano Heredia. Lima, Perú.

** Médico-residente de Neumología. Universidad Peruana Cayetano Heredia. Hospital Nacional Cayetano Heredia. Lima, Perú.

*** Jefe de la Unidad Cuidados Intensivos, Departamento de Medicina, Hospital Nacional Cayetano Heredia. Profesor Universidad Peruana Cayetano Heredia.

\& Jefe del Servicio de Neumología, Departamento de Medicina, Hospital Nacional Cayetano Heredia. Profesor Universidad Peruana Cayetano Heredia.
} 
Se presenta un caso de hidatidosis diseminada que causó un Síndrome de Vena Cava Superior, ambos hechos de presentación inusual según la revisión de la literatura.

\section{Caso Clínico}

Se trata de una mujer de 52 años, natural de Churín (zona rural al norte de Lima) y procedente del Japón donde vivió los últimos 8 años, llegando al Perú 15 días antes de su ingreso. Como antecedente era hipertensa de larga data, asmática y había sido operada de una "tumoración abdominal" quince años atrás.

Inició su enfermedad actual 4 horas antes de su admisión por emergencia, con cefalea fronto-parietal intensa, súbita, añadiéndose pérdida del conocimiento, convulsiones tónico-clónico generalizadas y relajación de esfínteres. La paciente estaba en coma, con mal patrón respiratorio (períodos de apnea), por lo que fue intubada y se inició ventilación mecánica.

El examen físico mostró una paciente en coma profundo, Glasgow 5, hemodinámicamente estable, pálida, con edema facial y de miembros superiores, circulación venosa colateral en cara anterior del tórax y abdomen a predominio derecho, telangiectasias, edema palpebral bilateral, quemosis, ingurgitación yugular presente, choque de punta cardiaco en $6^{\circ}$ espacio in- tercostal por fuera de la línea medio clavicular, soplo sistólico II/VI multifocal, roncantes escasos en bases pulmonares, abdomen levemente distendido, con cicatriz quirúrgica subcostal derecha y visceromegalia. Neurológicamente tenía signos de descerebración derecha y decorticación izquierda y reflejo de Babinsky bilateral (Figura $\mathrm{N}^{\circ} 1$ ).

La radiografía de tórax mostró un mediastino ensanchado, cardiomegalia global, lesiones cavitarias múltiples en tercio superior derecho, masa paramediastinal derecha e infiltrado alveolar basal del mismo lado (Figura $\mathrm{N}^{\circ} 2$ ). La tomografía computarizada (TC) cerebral mostró una imagen hiperdensa parietal posterior izquierda con marcado efecto de masa que desplazaba la línea media (Figura $\mathrm{N}^{\circ} 3$ ). En la TC de tórax se evidenció lesión cavitaria de paredes delgadas en tercio superior de hemitórax derecho, infiltrado alveolar e imágenes nodulares en base derecha, pequeño derrame pleural izquierdo y múltiples imágenes de aspecto quístico a nivel de mediastino, hígado y bazo (Figura $\mathrm{N}^{\circ} 4$ ). La ecografía abdominal y transtorácica mostraron quistes múltiples en hígado y mediastino.

La paciente ingresó a la Unidad de Cuidados Intensivos de Medicina (UCI), con el diagnóstico presuntivo de stroke hemorrágico e hipertensión endocraneana severa y síndrome de vena cava superior (SVCS) causado

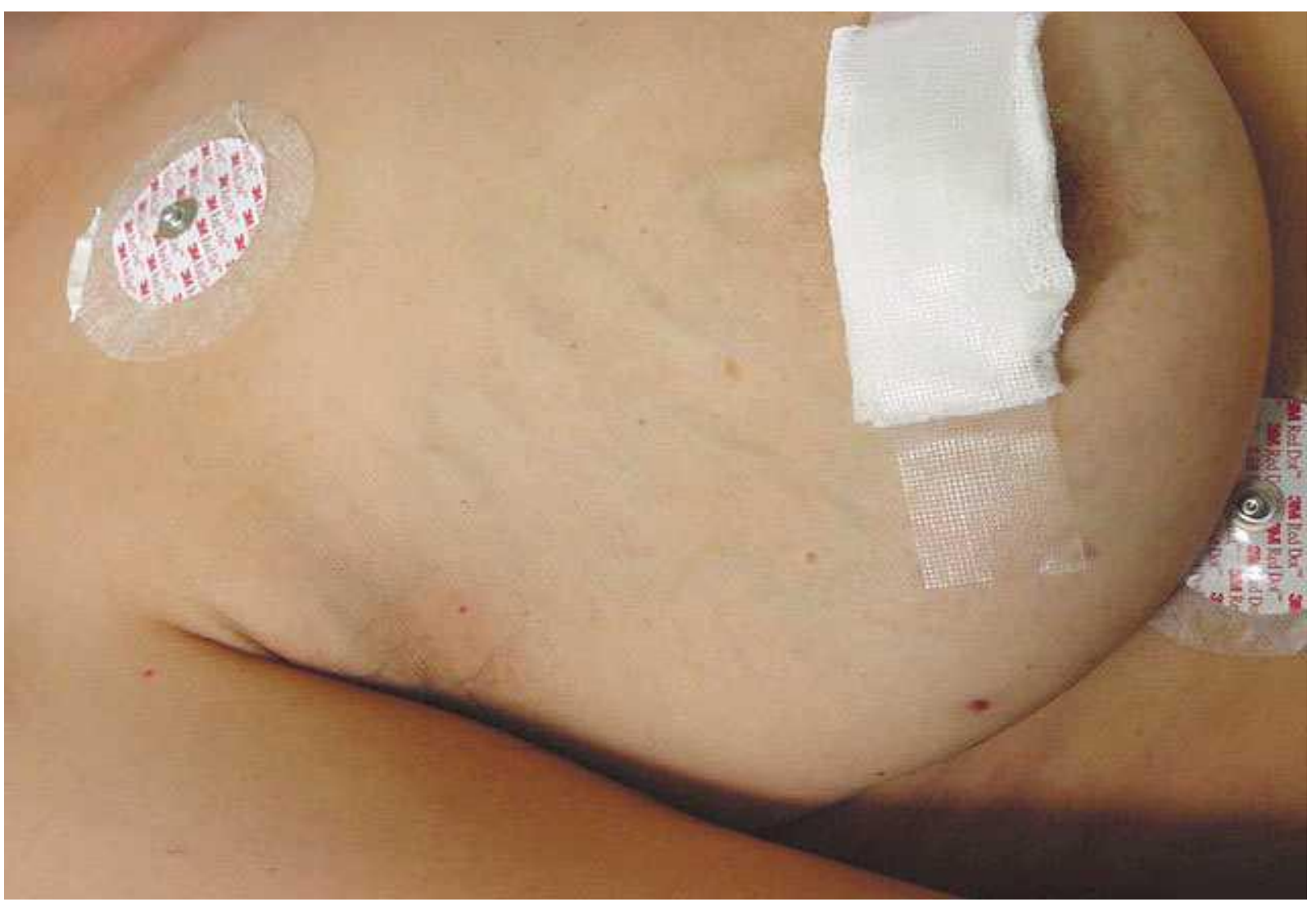

Figura №1 

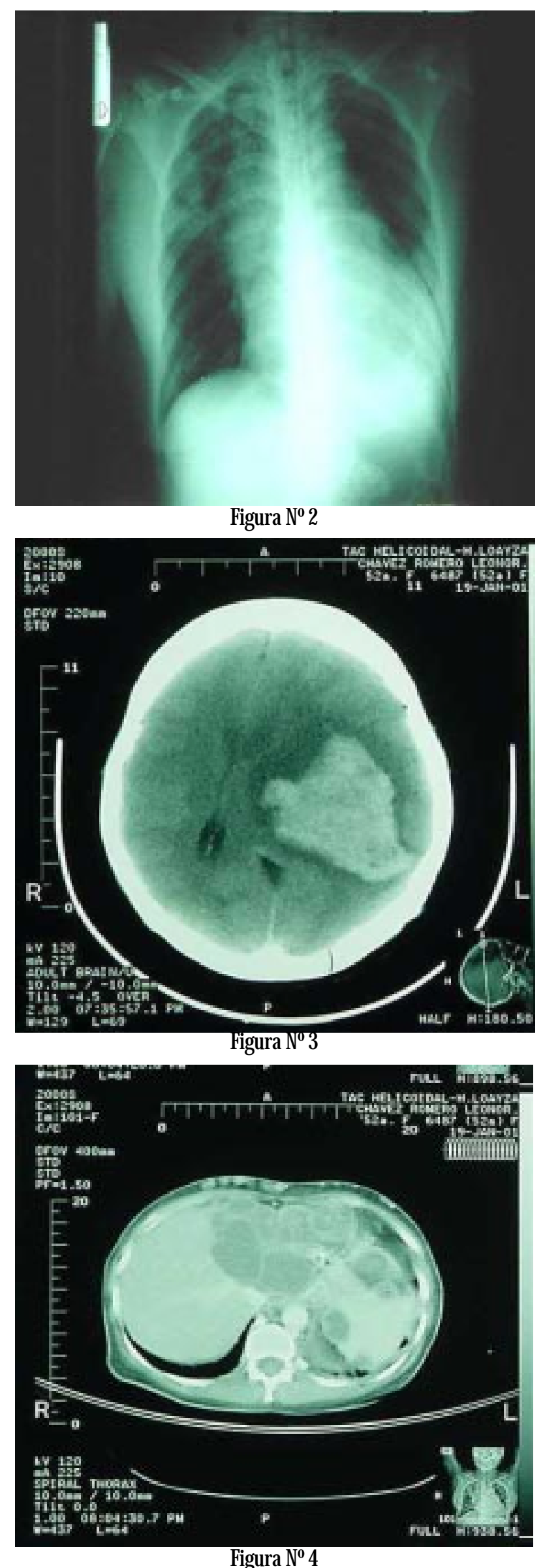

por masas a nivel mediastinal de etiología a determinar.

En el diagnóstico diferencial se incluía lesiones de mediastino anterosuperior con SVCS secundario, principalmente la posibilidad de neoplasia maligna que requiriese radioterapia de emergencia, como coriocarcinoma o tumores tiroideos, razón por el que se realizó estudios de dosaje de gonadotrofina coriónica humana (( HCG) y hormonas tiroideas, cuyos resultados fueron normales.

A la paciente se le realizó una biopsia percutánea por aspiración con aguja fina guiados por tomografía, cuyo informe de Patología reveló una membrana avascular de quiste hidatídico, resultando posteriormente la serología para hidatidosis (ELISA) positiva.

Debido al severo compromiso neurológico, se le inició tratamiento médico con Albendazol 400 mg bid, el cual se tuvo que descontinuar debido a una elevación de transaminasas séricas.

Luego de una semana en UCI, en donde estuvo en ventilación mecánica, la paciente salió de la unidad con traqueostomía. Posteriormente presentó neumonía nosocomial, falleciendo a los catorce días de su admisión, pese a recibir tratamiento antibiótico. La paciente en ningún momento se recuperó del compromiso neurológico.

La necropsia mostró macroscópicamente, lesiones quísticas múltiples a nivel pulmonar y mediastinal. El corazón tenía un pericardio engrosado y fibroso, con alteración morfológica por múltipes estructuras quísticas, lesiones similares en el lóbulo izquierdo hepático, bazo y mesenterio. En el SNC se encontró hemorragias intra y extraparenquimales, edema marcado con herniación transcingular y transcallosa del lado izquierdo y derecho (Figuras $\mathrm{N}^{\circ} 5$ y 6 ). Microscópicamente, se encontró en cerebro tejido nervioso con vacuolización (por hipertensión arterial), edema, necrosis, hemorragia y foco de inflamación aguda. Además, se encontraron lesiones con capa fibrosa y otra multilaminar, compatibles con quiste hidatídico, con células gigantes multinucleadas tipo cuerpo extraño, tanto a nivel pulmonar como en corazón, mediastino, hígado, bazo y mesenterio (Figuras $\mathrm{N}^{\circ} 7 \mathrm{y}$ 8).

Con esta información se concluyó que se trató de un caso de hidatidosis diseminada, con afección de mediastino, corazón, pulmón, hígado, bazo y mesenterio; con compresión externa de grandes vasos provocando un SVCS, que presentó hemorragia cerebral extensa de causa hipertensiva y herniación de amígdalas 

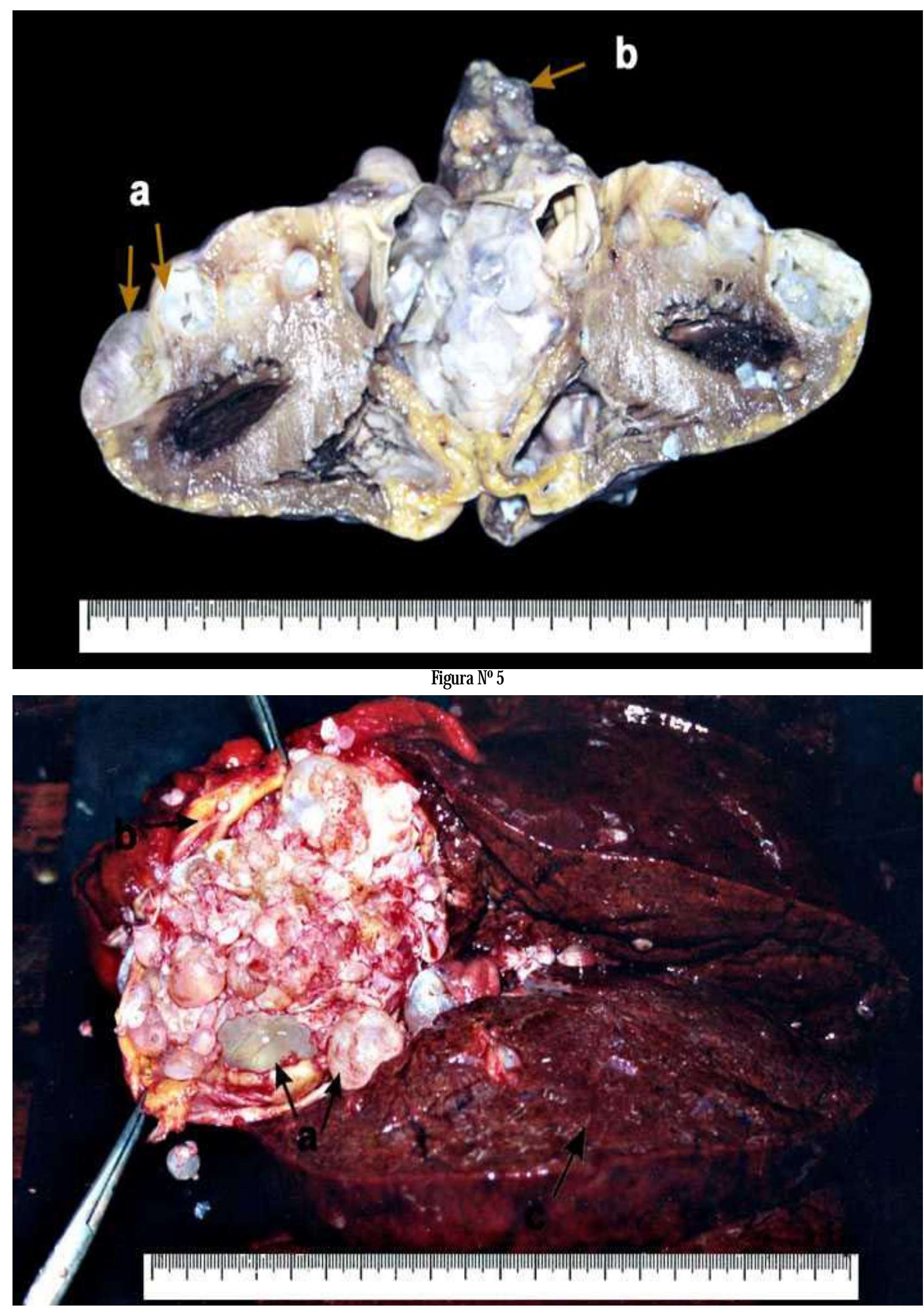


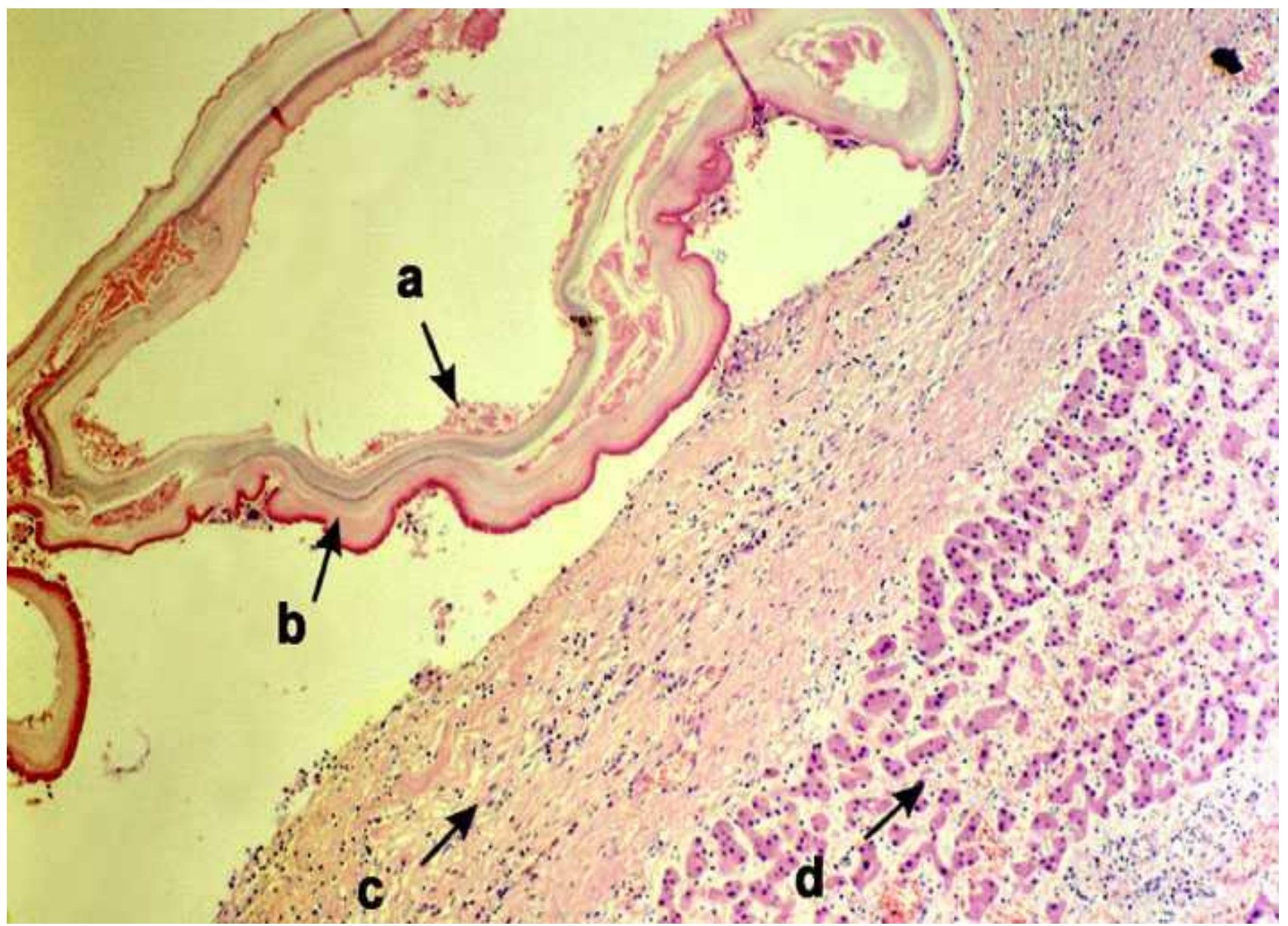

Figura № 7

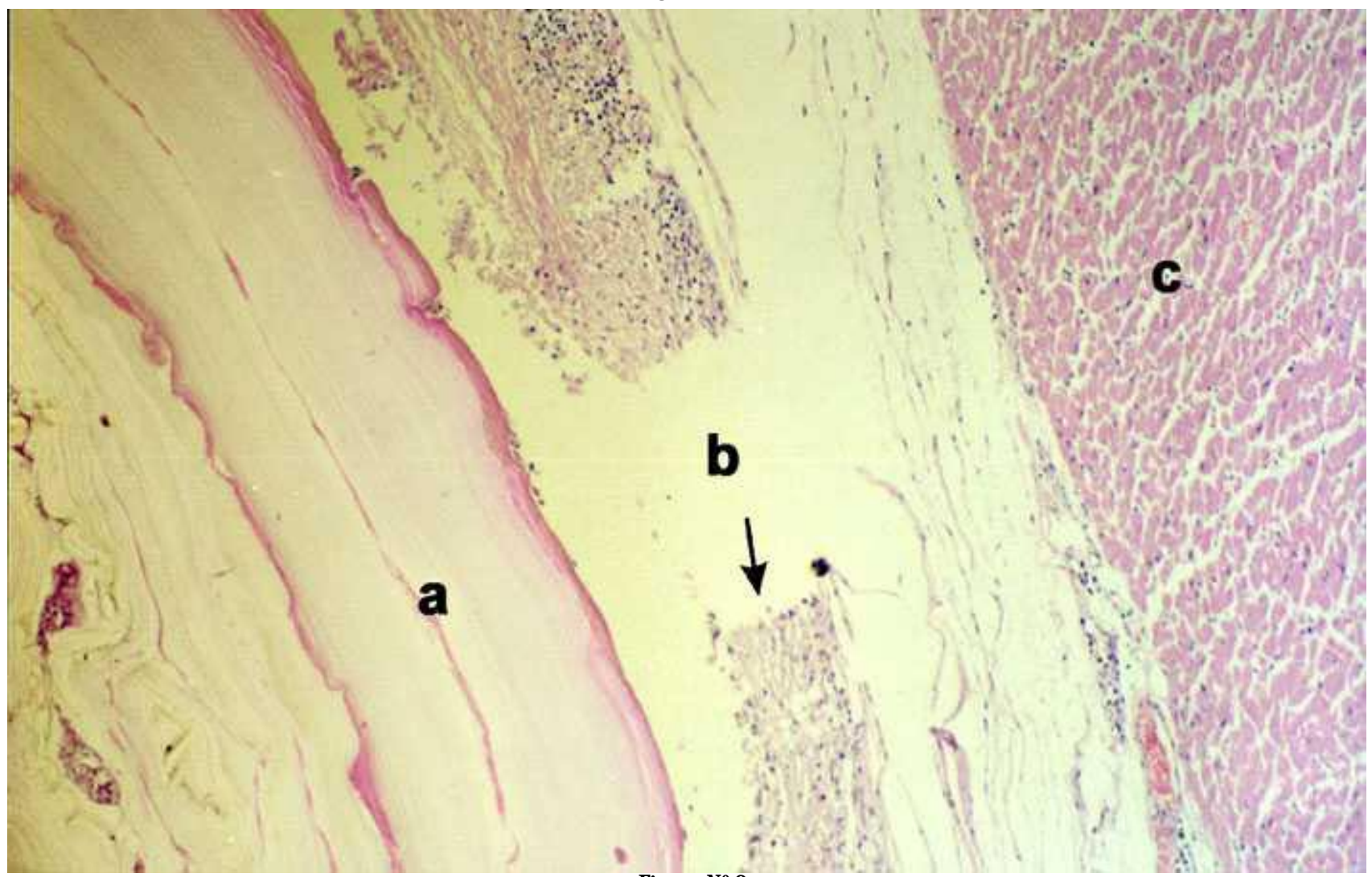


cerebelosas.

\section{DISCUSIÓN}

Los quistes hidatídicos se localizan frecuentemente en el hígado (60\%) y en los pulmones (25\%)(7). Otras localizaciones menos frecuentes, incluye la localización mediastinal que representa un $0.1 \%$ de todas las presentaciones y no excede el $1 \%$ de las localizaciones intratorácicas (4).

Esta enfermedad generalmente sólo compromete un órgano y en $13 \%$ de los casos se comprometen dos órganos. La presentación diseminada es poco frecuente; al respecto, un estudio realizado en Jordania, evaluó 306 casos de hidatidosis, encontrando un $7.5 \%$ con compromiso multiorgánico (5).

La ampliación de la anamnesis reveló que la paciente había presentado desde la infancia una tumoración abdominal en epigastrio de crecimiento lento, posterior a un traumatismo en dicha zona. Al inicio era indolora, progresivamente se añadieron episodios de coluria y dolor, siendo sometida a cirugía en 1983, en el hospital de su localidad. La historia clínica de dicha hospitalización señalaba en el reporte operatorio que el procedimiento incluyó liberación de adherencias, punción de la tumoración en 3 oportunidades, quistectomía y marsupialización. Se encontró un quiste hidatídico hepático gigante (contenía 4 litros de líquido, éscolex e hidátides hijas en cantidad) por debajo del lóbulo izquierdo del hígado con múltiples adherencias a epiplon y pared peritoneal y la paciente no recibió tratamiento médico posterior.

La paciente después de la operación, volvió a consultar por dolor abdominal en reiteradas ocasiones; en una de ellas inclusive con ictericia, pero no se llegó a establecer un diagnóstico preciso, llamando la atención la persistencia de "hepatomegalia" en ecografías posteriores y eosinofilia persistente.

La paciente ingresó a nuestro hospital por un accidente cerebrovascular de causa hipertensiva y circunstancialmente se descubren lesiones quísticas múltiples en diversos órganos, incluyendo mediastino anterosuperior que provocaban un síndrome de vena cava superior, con la triada clásica de edema en esclavina, cianosis de manos y a nivel facial y circulación colateral en tórax (6). Este síndrome se asocia principalmente a malignidad (85-97\%), entre ellos cáncer de pulmón (80\%), linfoma (10\%) y metástasis $(10-15 \%)$, aunque también se han descrito causas benignas (quistes, bocio, teratoma, timoma, procesos inflamatorios como mediastinitis crónica, fibrosis por radiación, tuberculosis, histoplasmosis, actinomicosis, aneurismas) además de los descritos en relación a trauma y catéteres centrales (5). Debe resaltarse que los síntomas no guardan correlación con la benignidad o malignidad de la lesión, pudiendo ambos tener el mismo cuadro (7).

En este caso la presencia de múltiples quistes de mediastino fue la causa del SVCS, hecho descrito en la literatura. Esta localización es rara, fue descrita por vez primera por Trigo en una serie de 74 casos en 1959. Posteriormente en 1960, Rakower y Mildwidsky describen otra serie de 78 casos. En 1989 Nin Vivo describe incidencia de 7/1815 tumores torácicos por quiste hidatídico mediastinal; al año 1991 sólo se han descrito 100 casos en la literatura, entre ellos el estudio de Heras de tipo retrospectivo que entre los años 197898 encuentra que 8 de 453 tumores de mediastino eran por quiste hidatídico. La incidencia es similar para ambos sexos, con un tiempo de evolución entre 1 mes y 8 años $(8,9)$. Benzarti, M. et al., en Túnez, describen un caso de quiste hidatídico primario calcificado a nivel mediastinal, adherido a grandes vasos, pero sin invadirlos ni comprometerlos, causando sólo parálisis diafragmática por compresión de nervio frénico y recurrente laríngeo (10).

Respecto a los tipos de diseminación se describe que puede ser primaria (luego de ingesta de huevos) o secundaria (por diseminación de focos primarios), ya sea por vía hematógena; por ruptura de quistes en peritoneo, pleura, árbol bronquial o biliar; en forma espontánea, quirúrgica o traumática o por vía directa (tránsito abdomino-torácico), además de diseminación por embolia parasítica pulmonar $(3,4)$. En este caso, la diseminación pudo haber ocurrido tanto por ruptura intraoperatoria del quiste inicial como por diseminación microscópica hacia otros tejidos al momento de la primera cirugía.

Salepsi B. et al, en Turquía, describen un caso de hidatidosis diseminada con localización pulmonar, intra-abdominal e intracardiaca, a nivel del ventrículo derecho, que recibió tratamiento médico con albendazol, sin obtenerse respuesta, excepto en los quistes intraabdominales. En este caso nunca se encontró quistes a nivel hepático luego de 13 años de seguimiento (11).

Se ha descrito casos de Equinococosis cardiaca con una incidencia de 0.5 a $2 \%$. La ecocardiografía transtorácica es de elección para el diagnóstico. Los síntomas referidos incluyen palpitaciones, disnea, dolor torácico atípico, pudiendo presentar complicaciones fatales, entre ellas ruptura del quiste, infarto del miocardio, ICC, taquicardia ventricular, disturbios de conducción, arresto cardíaco y embolismo pulmonar 
o sistémico (12).

En el electrocardiograma se puede encontrar inversión de la onda T (80\%), depresión del segmento ST (37\%), latidos ventriculares prematuros (42\%), bloqueo de rama completo o no (58\%), TPSV (10\%). La ecocardiografía puede mostrar quistes dentro del ventrículo izquierdo (52\%), septum (42\%), en ventrículo derecho $(31 \%)$, en pericardio $(10 \%)(17,18)$. En esta paciente se encontraron quistes intracardiacos por ecocardiografía que fueron posteriormente confirmados en la necropsia, encontrándose además depresión del segmento ST e inversión de la onda T en el ECG (13).

Si bien en esta paciente no se demostró la presencia de hidatidosis a nivel cerebral, el diagnóstico diferencial de la afección del sistema nervioso incluía esta posibilidad. Se ha señalado que la incidencia de Equinococosis cerebral es de 1-2\%, con una edad media de presentación de 13.4 años. El diagnóstico es por tomografía computada y la localización usual es supratentorial, (parietal) por embolismo de la arteria cerebral media. Loa quistes pueden ser únicos o múltiples (raro) $(14,15,16,17)$.

Existen reportes de casos de cardioembolismo con afección en SNC o de la arteria femoral $(18,19)$. En esta paciente no se demostró que hubieran quistes a nivel cerebral, sin embargo la paciente tenía una alta chance de fenómenos cardioembólicos dada la presencia de quistes intracardíacos (dentro de aurícula izquierda).

El SVCS conlleva la presencia de hipertensión endocraneana a la cual se sumó la causada por el stroke hemorrágico, lo que finalmente llevó a la muerte a la paciente.

En cuanto al tratamiento, éste es principalmente quirúrgico; que en este caso no pudo ser realizado, por el severo compromiso neurológico desarrollado. El tratamiento médico según recomendaciones de la OMS se reserva para aquellos casos con quistes múltiples e inoperables, luego de cirugía incompleta y para prevención de equinococosis secundaria luego de ruptura espontánea o traumática. Mebendazol y albendazol son las drogas usadas; la experiencia general indica que con el tratamiento los quistes desaparecen en $30 \%$ de pacientes, degeneran o reducen su tamaño en $30-50 \%$ y en $20-40 \%$ permanecen sin cambio. Albendazol es usado a $10-15 \mathrm{mg} / \mathrm{kg} / \mathrm{día}$ y es bien tolerado. Hay estudios sobre su efectividad en disminuir la viabilidad de los quistes hidatídicos hepáticos cuando se usa en el preoperatorio $(20,21,22)$. El tratamiento es dado continuamente por 6 meses o en cursos de 4 semanas con intervalos de 2 semanas entre cada ciclo. Nuestro paciente recibió tratamiento con albendazol por pocos días, pues luego se consideró que el riesgo de desarrollar una reacción anafiláctica fatal era alto, por lo cual se suspendió dicha medicación.

Desafortunadamente, el compromiso neurológico severo en esta paciente y la infección intrahospitalaria intercurrente no permitieron un mejor desenlace siendo la evolución tórpida pese al manejo instalado.

\section{Correspondencia:}

Dr. José Luis Pinto Valdivia

Av. Honorio Delgado 235 4to piso, S.M.P.

Teléfono: 3826940 (casa), 8518145 (celular)

E-mail: josepinto99@medscape.com

\section{REFERENCIAS BIBLIOGRAFICAS}

1. García L. et al. Diagnostic medical parasitology. Second edition. American Society for Microbiology. Washington DC. 1991.

2. García EJ. Evaluación de los criterios clínicos y serológicos de la hidatidosis humana en Lima. Tesis de Grado de Bachiller en Medicina. Universidad Peruana Cayetano Heredia. Lima, Perú. 1991.

3. Atías A. Parasitología clínica. Tercera edición. Editorial Mediterráneo. Santiago de Chile. 1991.

4. Ammann RW, Eckert J. Parasitic diseases of the liver and intestines. Cestodes. Echinococcus. Gastr Clinics 1996; 25: 655-689.

5. Amr SS. et al. Hidatidosis in Jordan: An epidemiological study of 306 cases. Ann Trop Med Parasitol 1988; 6: 623-27.

6. Surós J. Semiología médica y Técnica exploratoria. Salvat Editores. 1972.

7. Schindler N. et al. Superior vena cava syndrome. Experience whit endovascular stents and surgical therapy. Surg Clin North Am 1979; 3: 683-94.

8. Heras F, Ramos G, Duque JL, García Yuste M, Cerezal RJ, Matilla JM. Quistes Hidatídicos de Mediastino: 8 casos. Arch Bronconeumol 2000; 36: 221-224

9. Jiménes Merchán R. Quiste Hidatídico de Mediastino. Arch Bronconeumol 1999; 35: 51-2

10.Benzarti M. et al. Kyste hydatique médiastinal primitif compressif calcifié. Rev Mal Resp 1997; 14: 53-54.

11.Salepsi B. et al. Disseminated hydatid disease with intracardiac localization: A case report. Clin Pulm Med 2000; 7(6): 304-307.

12.Atilgan D, Demirel S, Akkaya V, Korkut F. Left ventricular Hydatid Cyst: An unusual location of Echinococcus Granulosus with multiple organ involvement. J Am Soc Echocardiogr 1996; 9:212-5

13.Bashour T. Echinococcosis of the heart: Clinical and ecocardiographic features in 19 patients. Am Heart J 1996; 132:1028-30.

14.Gupta S. Intracranial Hydatid Cyst: A report of five cases. Neurol India 1999; 47:214-7. 
15.Gezen F. et al. Hydatid cysts of the brain. Clin Infect Dis 1995; 21: 9938-42.

16.Aguirre I. et al. Quiste hidatídico cerebral en niños en el Hospital Nacional Cayetano Heredia. A propósito de un caso. Rev Med Hered 1993; 4:155-57.

17.Abdulla K. et al. Ruptured cerebral hydatid cyst: A case report. J Trop Med Hyg 1988; 9:302-305.

18.Turgut M..et al. Secondary multiple hydatid cysts caused by intracerebralembolism of cardiac echinococcosis: an exceptional case of hydatidosis. Case report. J Neurosurg 1997; 86:714-8.
19.Quesar E. et al. Cerebral vascular accidents due to hydatid embolisms. J Neuroradiol 1996; 23:74-8.

20.Nutman TB. et al. Infectious diseases: Cestodes. In: Fauci AS, Braunwald E, Isselbacher KJ, et al., editors. Harrison's Principles of Internal Medicine. New York. McGraw Hill. 1988. pp: 1225-6.

21.Saimot AG. et al. Albendazole as a potential treatment for human hydatidosis. Lancet 1983; 2: 652-6.

22.Aktan AO. et al. Preoperative albendazole treatment for liver hydatid disease decreases the viability of the cyst. Eur J Gastroenterol Hepatol 1996; 8: 877-9. 